\title{
Inhibition of learned-response availability: Reduction of cued retrieval by frequency of occurrence and prior recall of target words
}

\author{
MELVIN H. MARX \\ University of Missouri, Columbia, Missouri \\ and \\ YUNG CHE KIM \\ Keimyung University, Daegu, South Korea
}

This experiment attempted to replicate Marx's (1982) report that transfer (anagram solutions) was inhibited both by more frequent occurrence of target words in a word-completion exercise and by the prior recall of the target words. We substantially confirmed both of these inhibitory factors, but especially the prior recall: Target words that had occurred only once but had been recalled were subsequently retrieved relatively more often than were singly occurring but unrecalled words by a mere $1 \%(5 / 342)$ of the subjects. Other reliable main effects and interactions were found for the time intervals before free recall and before cued-retrieval testing and for the word-frequency variable. Interpretation of the inhibitory effect of prior recall on learnedresponse availability was tentatively made in terms of a Zeigarnik-like function.

Marx (1982) reported that target words that had been completed in a word-completion exercise by high school students and subsequently recalled were less likely to be solved as anagrams in a final transfer test than were target words that had been completed but not recalled. There was also an inverse relationship between frequency of target-word occurrence and transfer scores.

The present study was planned to replicate these somewhat surprising, counterintuitive results. Marx (1982) had used two-word anagrams consisting of common English phrases (e.g., hot dog, snow fall, flat tire) after one of the words had been used with varying frequency in the word-completion test, which was followed immediately by a free-recall test. The first letter of each word was provided (e.g., h__ d__ ) to facilitate anagram solution in the restricted time period allowed. Two-word combinations with scrambled letters were also used in the present experiment, with one word in each pair having served as a target in the prior word-completion exercise. However, the words were unrelated, and, more importantly, each old target word was cued by one of the same short phrases that had been used in the wordcompletion exercise. Thus, the present study is con-

This research was supported in part by a Research Career Award from the National Institute of Mental Health to the first author. The second author was responsible for the collection and primary statistical analysis of the data in Korea. M. H. Marx's mailing address is: Department of Psychology, University of Missouri, Columbia, Missouri 65211. Yung Che Kim's mailing address is: Department of Psychology, Keimyung University, Daegu, South Korea. cerned with a kind of cued retrieval rather than with transfer. Nevertheless, because of the close and interlocking relationships among the various forms of retrieval, it seems reasonable to assume that there is a high degree of commonality in the underlying principles that determine the availability of previously established ("learned") responses.

\section{METHOD}

Subjects

A total of 75211 th-grade students in two large senior high schools in Daegu, South Korea, served as subjects. They were tested in their regular classes, which were assigned randomly to the various treatment groups.

Design

The basic design was a mixed factorial analysis of variance (ANOVA) with one within-subjects variable ( 3 levels of frequency of occurrence of target words) and gender (2) $x$ performance or observation (2) $x$ free-recall delay (3) $x$ retrieval delay (3) between-subjects variables. ${ }^{1}$ In order to equate cell size of the resulting 36 groups at 19 subjects, 68 subjects were eliminated randomly from the larger classes. Males and females were in separate classes.

\section{Materials}

In the word-completion task, 24 words were repeated one, two, or three times, providing a total of 48 word items, randomly ordered, to be completed. Each word had three or four letters, only one of which was given. Cues (associated meanings) were provided for all of the items (e.g., largest sea in the world, for Pacific; plaything, for toy; deceive, for lie). Different cues were used in the repetitions of target words, with frequencies of 2 (F2) and 3 (F3); examples of the cues used were: drag-net, work in the ocean, and catching for the target word fishing; 
underground traffic, underground railroad, and traffic in big city for the target word subway. The retrieval test comprised anagram-like letter strings, with each string capable of being broken down into two words. One of the two words had been used in the word-completion task. Each of the two words was cued by phrases of the same sort as had been used in the wordcompletion task. For the "old" word, that is, the previously used one, an old cue was used.

All materials and instructions were in Korean script. The word-completion task was presented on 7 pages, each approximately $20 \times 27 \mathrm{~cm}$, in booklet form; the retrieval test was presented in 4 similar pages. In the absence of any published word-frequency norms, word frequencies were equated, in the preparation of the lists, by word counts made in basic textbooks published by the Korean Ministry of Education for Grades 1 through 6. Examples were provided with the instructions for each task.

\section{Procedure}

Word-completion task. Students in each class were tested in pairs. One student in each pair performed (that is, actually made the responses) while the other student observed, in a manner previously used (e.g., Marx, Homer, \& Marx, 1980). Performers paced themselves and were allowed $5 \mathrm{~min}$ for the exercise.

Free recall. A free-recall test was given after 0 (immediately), 1 , or 7 days. The students were allowed $2 \mathrm{~min}$ in which to recall as many of the previously completed words as they could. They worked individually in this and the subsequent retrieval test.

Retrieval. The cued-retrieval test was administered 0 (immediately), 1, or 7 days after the free-recall test. The students were given $5 \mathrm{~min}$ in which to solve as many of the words as they could from the letter strings.

\section{RESULTS}

The analyses reported below used only the 342 performance subjects-that is, the subjects who had made the actual responses during the word-completion exercise. ${ }^{1}$ Two different statistical techniques were used. The results of standard ANOVAs are reported first. The second kind of analysis was by chi-square tests of subject frequencies in different categories. This was considered more appropriate for the recall/no-recall category that was the primary concern of the experiment.

\section{Frequency of Occurrence}

In all of the following analyses, the word-completion frequency measure is based on the words that were actually completed by the subject at the assigned frequency value.

The mean numbers of words retrieved were 3.60 , 4.59, and 3.47 for $F 1, F 2$, and F3, respectively. These means are reliably different $[\mathrm{F}(2,666)=99.68, \mathrm{MSe}=$ $1.38, \mathrm{p}<.01]$.

Figure 1 presents these data in comparison with the free-recall mean scores, which show the usual positive relationship between frequency of response and recall.

\section{Test Delay}

The mean retrieval scores across the various testdelay groups are shown in Figure 2. ANOVA of the two delay variables indicated that both were effective determinants of retrieval $[\mathrm{F}(2,33)=4.22, \mathrm{MSe}=4.80$, $\mathrm{p}<.01$, and $\mathrm{F}(2,333)=6.44, \mathrm{MSe}=4.80, \mathrm{p}<.01$, for

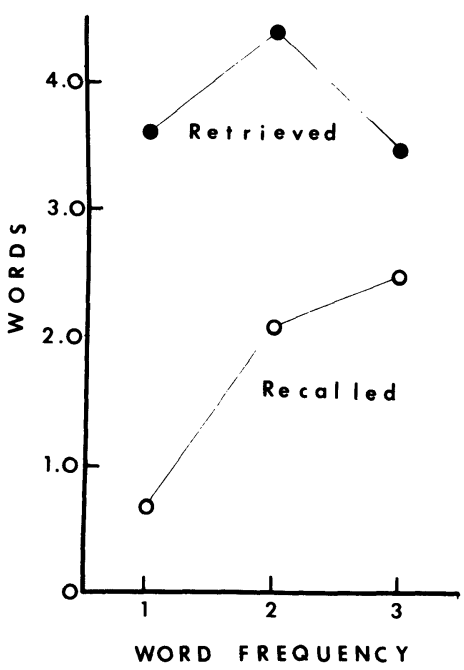

Figure 1. Mean words recalled/retrieved as a function of word frequency.

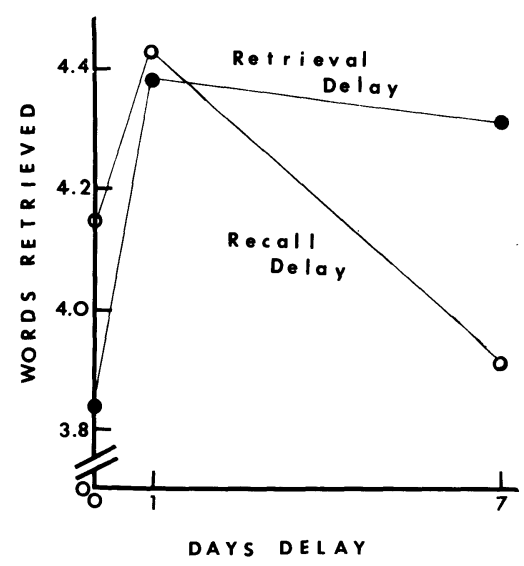

Figure 2. Mean words retrieved as a function of free recall and cued-retrieval test delays.

the free-recall and retrieval test delays, respectively]. In each case, the highest level of retrieval was for the 1-day-delay condition.

\section{Completed vs. Uncompleted Words}

Our first frequency analysis is of the proportions of subjects who retrieved a higher percentage of words that they had previously completed during the wordcompletion exercise. Exactly half of the subjects (171 of 342 ) retrieved relatively more completed than uncompleted F1 words, 69\% (237) did so for F2 words, and $42 \%$ (144) did so for F3 words. If the overall proportion $(552 / 1026=.538)$ is taken as a theoretically expected baseline, the $F 1$ proportion is not reliably different $\left[\chi^{2}(1)=1.99, p>.05\right]$, but both the higher proportion for F2 words and the lower proportion for F3 words are reliably different $\left[\chi^{2}(1)=33.05, p<.01\right.$, for $F 2$ words, and $\chi^{2}(1)=18.83, p<.01$, for F3 words] .

In order to simplify the analysis and yet utilize all of 
the data, we combined the two delay intervals into a single delay measure, the total number of days between the initial word-completion exercise and the final retrieval test. This combination produced total day delays of 0 (the 0 -day free-recall and 0 -day retrieval test delays), 1 (the 0-1- and 1-0-days groups, for free-recall and retrieval test delays), 2 (the $1-1$ group), 7 (the 0.7 and $7-0$ groups), 8 (the $1-7$ and $7-1$ groups), and 14 (the 7-7 group).

Because of the clear break between short (0-, 1-, and 2-day) and long (7-, 8-, and 14-day) delays, the three short-delay conditions were then combined and compared with the similarly combined long-delay conditions. Reliably more subjects retrieved higher proportions of F1 and F2 completed words in the short- than in the long-delay conditions: $77 / 152=51 \%$ compared with $74 / 190=39 \%$ for $F 1$ words $\left[\chi^{2}(1)=4.10, p<.05\right]$ and $114 / 152=75 \%$ compared with $123 / 190=65 \%$ for F2 words $\left[\chi^{2}(1)=9.11, p<.01\right]$ for the short and long delays, respectively, but there was no difference for the F3 words: $61 / 152=40 \%$ compared with $83 / 190$ $=44 \%\left[\chi^{2}(1)=.43, \mathrm{p}>.05\right]$.

\section{Prior Recall}

The most surprising result of this study occurred when we examined the proportion of subjects who retrieved relatively more of the completed words that they previously had recalled than of those that they had previously failed to recall. Figure 3 shows retrieval as a function of both the frequency-of-occurrence variable and the six total test-delay periods. Apparently, a single occurrence, when recalled, had an extremely powerful inhibitory effect on subsequent retrieval. The total of 5 of 342 such subjects (1\%) for the F1 condition contrasts sharply with the total of 89 such subjects (26\%) for the F2 condition and 224 such (65\%) for the F3 condition. If the overall proportion $(413 / 1026=.306)$ is taken as a theoretically expected baseline, the lower

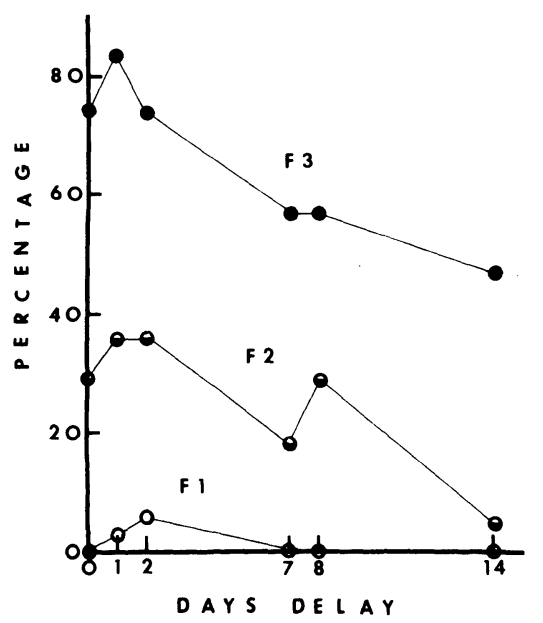

Figure 3. Percentage of subjects who retrieved a higher proportion of completed-recalled than completed-nonrecalled words for the three word frequencies.
F1 proportion is quite reliably different $\left[\chi^{2}(1)=136.83\right.$, $\mathrm{p}<.01]$, as is the higher proportion for F3 words $\left[\chi^{2}(1)=195.92, p<.01\right]$. The proportion for F2 words is not reliably different $\left[\chi^{2}(1)=3.39, \mathrm{p}>.05\right]$.

Figure 3 also shows the strong influence of the delay variable on the prior-recall factor. Although the extremely small proportions for the F1 words were not reliably different $\left[\chi^{2}(1)=2.67, p>.05\right.$, for the $4 / 152$ representing short delays compared with 1/190 representing long delays], the corresponding test-delay proportions for F2 $(51 / 152=30 \%$ vs. $38 / 190=20 \%$ for short and long delays, respectively) and for F3 (120/152 $=79 \%$ vs. $104 / 190=55 \%$, again for short and long delays, respectively) were both reliably different $\left[\chi^{2}(1)=\right.$ $8.08, \mathrm{p}<.01$, for $\mathrm{F} 2$, and $\chi^{2}(1)=21.81, \mathrm{p}<.01$, for $F 3$ ]

\section{DISCUSSION}

Both of the counterintuitive results reported by Marx (1982) are supported by the analyses in this study. The inhibitory effect on subsequent retrieval of prior recall of a word completed one time is especially striking. Also noteworthy is the fact that this strong F1 inhibitory effect was essentially uniform across the delay variable, rather than being restricted to the longer delays, as happened for the F2 and the F3 conditions (cf. Figure 3; however, note also the extremely low percentage of F2 targetword retrieval after the full 14-day delay).

We conclude that the inhibitory effect of prior recall on transfer reported by Marx (1982) is also characteristic, at least for small frequencies of occurrence and for relatively long delays before testing, for the kind of cued retrieval that was used in the present experiment.

Some confirmation, although much less striking, is also evident for the inhibitory effect of the frequency-of-occurrence variable that Marx (1982) reported. The inverted curve representing the relationship between frequency of occurrence and retrieval of target words (Figure 1) is similar to the inverted curves reported by Marx (1982, Figure 2), although in the latter case the highest retrieval levels were found for the F1 rather than the $\mathrm{F} 2$ words. In the present data, there is a sharp contrast with the orthodox linear dependence of free-recall scores on frequency of occurrence (Figure 1).

It is interesting that this inverted relationship between frequency and retrieval variables did not appear when the retrieval measure was the proportion of subjects retrieving higher percentages of recalled than of nonrecalled words (Figure 3). By that measure, only the $\mathrm{F} 1$ frequency level and the longer test delays at the F2 level showed an inhibitory effect. There was, however, another inverted relationship between retrieval and word frequency. The proportion of subjects retrieving relatively more completed than uncompleted words was higher for F2 and lower for F3 words (with no difference in F1 words). The fact that the inversion occurred for both of these response-strength measures but not for the proportion of recalled responses is consistent with the presumption that the inhibitory factor primarily operates on less well established responses.

The present results not only offer considerable support to the inhibitory effects of frequency and prior recall but also extend the effect to the test-delay variable. That superior retrieval should occur, for each of the test delays, after 1 day rather than after no delay is an interesting result in its own right, even if not readily interpretable. The influence of the delay variable on availability of response is more directly suggested by the reliable differences in proportions of subjects retrieving relatively more completed than uncompleted $F 1$ and F2 words after short and long delays; only with more securely established F3 words were subjects as successful in utilizing completed words in retrieval after long delays. 
A similar kind of relationship between short and long delay intervals is evident for the proportions of subjects who retrieved more recalled than nonrecalled words. Higher proportions after short delays for both F2 and F3 words again suggest the weakening with time of the strength of the completed words.

What is responsible for the evident restriction of response availability under the conditions of the previous and the present research? The present data strongly suggest that a single emission of a weakly established response (i.e., recall of F1 word) severely restricts the subsequent availability of that response, especially when compared with the effect of a single emission of a more strongly established response (i.e., recall of F3 word), and particularly after long delay intervals (cf. Figure 3). The previous suggestion (Marx, 1982) of a Zeigarniklike function (Zeigarnik, 1927) may be developed, at least in a preliminary manner. If the initial emission of a response in a specific task situation induces some amount of motivational charge in that response-that is, a kind of increased potential to discharge, so to speak-then even a single such discharge, as in free recall, would be expected to return the response to its original low potential and thereby reduce its availability in the same general framework (i.e., class experimental situation). Although this sketchy outline is in some ways simply a rewording of what we have observed, it may help to conceptualize the phenomenon in a kind of framework that makes it susceptible to appropriately conceived research probes.

Our concern in this research has been with what we might call a kind of "incidental retention." Our primary interest concerns the conditions under which learned responses are utilized when direct, intentional recall is not involved. All of the manipulations that we have reported are related to that objective. Delineation of the fundamental principles that determine the availability of learned responses under such indirect retrieval tests is an important problem that has been largely neglected.
Nevertheless, this kind of utilization of learned responses seems ecologically significant and, as a complement to straightforward retention tests, poses a challenging theoretical problem.

Viewed from this general perspective, the primary research issue spurred by the present results is not only "Why did they occur?", but also "What are the principles that determine response availability under indirect retrieval conditions?" We hope that the present report will help to stimulate research bearing on both of these questions.

\section{REFERENCES}

Marx, M. H. (1982). Effects of frequency of prior incidental occurrence and recall of target words on anagram solution. Bulletin of the Psychonomic Society, 19, 253-255.

MarX, M. H., Homer, A. L., \& MARX, K. (1980). Verbal discrimination learning and retention as a function of task and performance or observation. Bulletin of the Psychonomic Society, 15, 167-170.

ZEIGARNIK, B. (1927). Das behalten erledigter und unerledigter handlungen. Psychologische Forschung, 9, 1-85. [Translated and condensed as "On finished and unfinished tasks" in W. D. Ellis (Ed.), A source book of Gestalt psychology. New York: Harcourt, Brace and World, 1983.]

\section{NOTE}

1. The gender and performance/observation variables and their interactions are treated separately, as are most of the results of the free-recall test, which was necessary for the present experiment but incidental to our primary objectives.

(Manuscript received for publication October 24, 1983.) 